\title{
Reliability of aortic MRI at 3 Tesla in patients with acute cryptogenic stroke
}

\author{
A Harloff, ${ }^{1}$ P Dudler, ${ }^{1}$ A Frydrychowicz, ${ }^{2}$ C Strecker, ${ }^{1}$ A L Stroh, ${ }^{3}$ A Geibel, ${ }^{3}$ C Weiller, ${ }^{1}$ \\ A Hetzel, ${ }^{1} \mathrm{~J} \mathrm{Hennig}^{2}{ }^{2}$ M Markl ${ }^{2}$
}

\section{See Editorial Commentary, p 489}

${ }^{1}$ Department of Neurology and Clinical Neurophysiology, University Hospital Freiburg Freiburg, Germany; ${ }^{2}$ Department of Diagnostic Radiology, Medical Physics, University Hospital Freiburg, Freiburg, Germany; ${ }^{3}$ Department of Cardiology and Angiology, University Hospital Freiburg, Freiburg, Germany

Correspondence to: A Harloff, Department of Neurology and Clinical Neurophysiology, University of Freiburg, Breisacher Str 64, D-79106 Freiburg, Germany; andreas.harloff@ uniklinik-freiburg.de

Received 18 May 2007 Revised 10 October 2007 Accepted 11 October 2007

Published Online First

26 October 2007

\section{ABSTRACT}

Objectives: To evaluate a new three dimensional (3D) MRI protocol for the reliable detection of aortic high risk plaques compared with transoesophageal echocardiography (TOE) and to test the reliability of additional MRI in stroke of undetermined aetiology.

Methods: 74 acute stroke patients were examined by both TOE and MRI at 3 Tesla with special regard to aortic high risk plaques (ie, $\geqslant 4 \mathrm{~mm}$, superimposed thrombi). ECG synchronised pre- and post-contrast T1 weighted 3D imaging (spatial resolution $\sim 1 \mathrm{~mm}^{3}$ ) covering the thoracic aorta was employed. In plaques $\geqslant 3 \mathrm{~mm}$, additional two dimensional T2 imaging and time resolved (CINE) imaging sequences were performed. Aetiology of brain ischaemia was classified according to modified TOAST (Trial of Org 10172 in Acute Stroke Treatment) criteria. Aortic high risk embolic sources detected by MRI in patients with cryptogenic stroke were evaluated.

Results: Differences in maximum aortic wall thickness for TOE and MRI were not statistically significant for all aortic segments. The overall number of high risk plaques detected by MRI $(n=74)$ was substantially higher compared with TOE $(n=47)$. Most noticeably, MRI identified aortic high risk pathologies in 8/26 (30.8\%) patients with cryptogenic stroke after standard diagnostics, including TOE $(n=2$ : dissection or thrombus; $n=6$ : plaques $\geqslant 4 \mathrm{~mm}$ ).

Conclusions: Our results demonstrate the feasibility of this 3D MRI protocol for the reliable detection of aortic high risk plaques in acute stroke patients. Because of improved visualisation of the aortic arch and the detection of additional embolic sources not seen by standard diagnostics, this novel technique may become a valuable tool for future patients with cryptogenic stroke.

Transoesophageal echocardiography (TOE) is the current reference standard for the detection of cardiac high risk sources of cerebral embolism, such as aortic plaques $\geqslant 4 \mathrm{~mm}$ and aortic thrombi. ${ }^{1}$ In patients with stroke of undetermined aetiology, these pathologies are by far the most frequent cardiac high risk sources detected by $\mathrm{TOE}^{2}$ and their prevalence is comparable with that of carotid artery stenosis and atrial fibrillation. ${ }^{3}$ Although the complication rate during $\mathrm{TOE}$ is low, ${ }^{4}$ the procedure is semi-invasive. Furthermore, visualisation of both the distal ascending aorta and aortic arch is strongly reduced by air artefacts and insonation angles. Thus diagnostic accuracy is limited and the exact number of embolic sources overlooked by TOE is currently unclear. ${ }^{3}$

Previous MRI studies on detection of atherosclerotic plaque were conducted on 1.5 Tesla systems only, and were limited with respect to spatial resolution, anatomical coverage and signalto-noise ratio ${ }^{5-7}$ while $3 \mathrm{~T}$ MRI improved characterisation of plaques ex vivo in a recent study. ${ }^{8}$ Also, these studies focused on the detection and composition of plaques either in the descending aorta or in the carotid arteries comparing MRI with TOE or histopathological examination..$^{9-13}$ However, in patients with cardioembolic stroke, the aortic arch (ie, the origin of the brain supplying arteries) is of high clinical interest. MRI of this region is still challenging as examination is strongly hampered by artefacts due to vessel motion and respiration.

In general, previously reported two dimensional (2D) MR studies suffered from incomplete coverage of the aortic segment of interest. For reliable acquisition of the aortic arch, the use of a dedicated TOE-MRI coil was reported. ${ }^{11-14}$ However, reduced patient comfort and limited availability of TOEMRI coils restrict clinical application to experimental studies. More conventional MR imaging using 2D slices positioned perpendicular to the curved aortic arch was successfully performed in animal models of induced atherosclerosis and in a recent patient study. ${ }^{15-17}$ However, high resolution MRI at $3 \mathrm{~T}$ for the detection of atheroma with three dimensional (3D) coverage of the thoracic aorta has not been reported to date. Also, the reliability of additional aortic MRI has not yet been investigated systematically in acute stroke patients.

The aim of this study was therefore to test the agreement between $3 \mathrm{D}$ MRI at $3 \mathrm{~T}$ and $\mathrm{TOE}$. Furthermore, we hypothesised that because of the improved visualisation of the aortic arch and the detailed retrospective data evaluation, MRI would be equivalent or even superior to TOE in detecting aortic pathologies, in particular in patients with cryptogenic stroke despite extensive clinical routine diagnostics.

\section{PATIENTS AND METHODS \\ Study population}

Seventy-four non-consecutive patients hospitalised in our stroke unit fulfilled the inclusion criteria: age 17-85 years, acute brain ischaemia, performance of TOE examination, no contraindications to $3 \mathrm{~T}$ MRI examination. The prospective study was approved by the local ethics committee and written informed consent was obtained from all participants. Following recommendations of a previous study, ${ }^{2}$ TOE was scheduled if routine diagnostics (brain MRI including MR angiography or CT, or both, transthoracic echocardiography, ECG, extra-/intracranial ultrasound) could not 

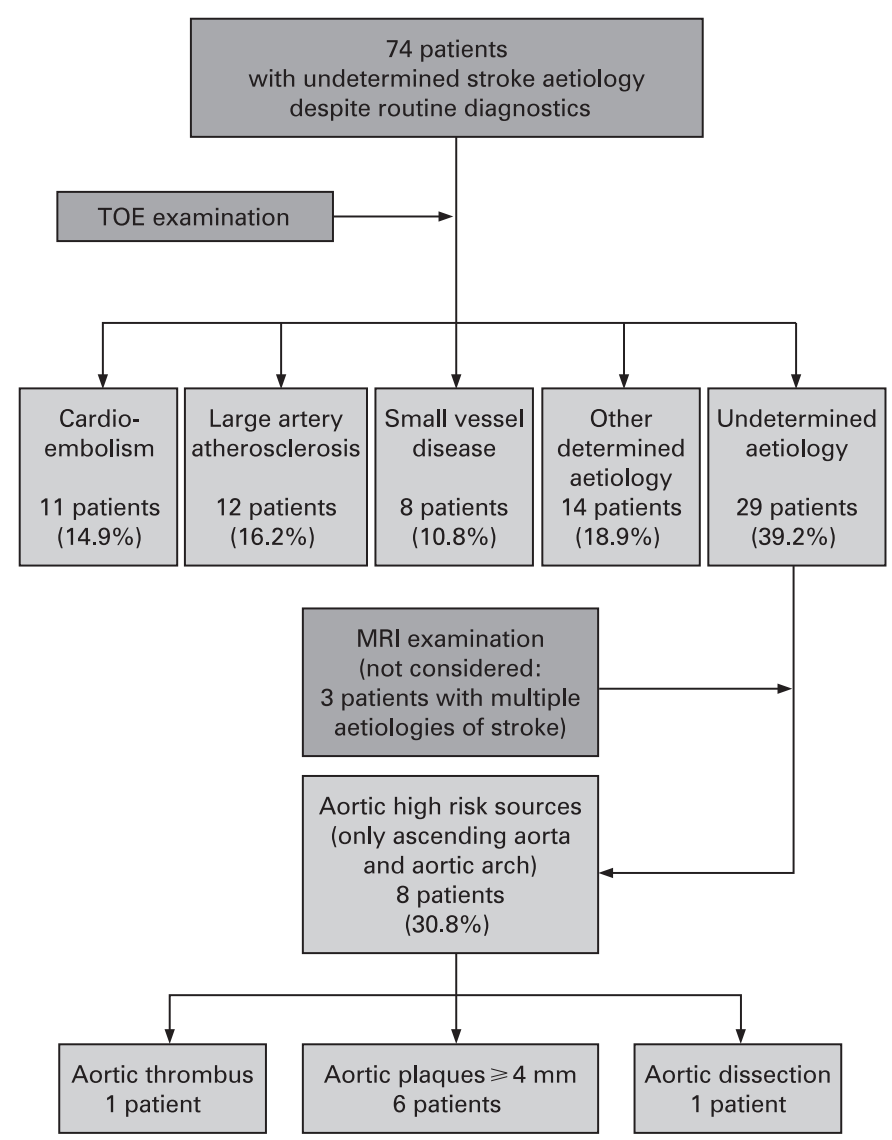

Figure 1 Distribution of stroke subtypes within the study population according to the TOAST (Trial of Org 10172 in Acute Stroke Treatment) criteria, and additional findings of MRI in patients with stroke of undetermined aetiology. TOE, transoesophageal echocardiography.

clarify stroke aetiology with a high level of confidence. In addition, TOE was performed individually in younger patients to screen for other cardiac sources, in patients with internal carotid artery stenosis, to exclude an additional high risk embolic source such as complex aortic plaques, and in patients with a history of atrial fibrillation but sinus rhythm in the actual Holter-ECG. Patients underwent both TOE and MRI examinations within 5 and 6 days (median) after admittance to our hospital. Median time interval between TOE and MRI examinations was 1 day.

Infarct aetiology was classified according to modified TOAST (Trial of Org 10172 in Acute Stroke Treatment) criteria ${ }^{18}$ taking the results of both routine diagnostics and TOE into account. With respect to additional TOE findings, stroke was classified as "cardioembolic" if left atrial appendage thrombus, spontaneous echo contrast or left atrial appendage flow velocity $<30 \mathrm{~cm} / \mathrm{s}$ was found. Stroke was defined as "other determined aetiology" if aortic plaques $\geqslant 4 \mathrm{~mm}$, aortic thrombi or dissection was detected by TOE. Patients with two or more probable sources of stroke were defined as "determined". Thus "undetermined" (ie, cryptogenic) stroke was reserved for those without a clarified aetiology despite complete diagnostics, and aortic high risk sources additionally detected by MRI in the ascending aorta or the aortic arch were further evaluated in this subgroup (fig 1). Embolic brain ischaemia was defined as present in patients with a typical pattern on brain imaging (ie, grey matter involvement: disturbed diffusion weighted imaging on MRI or hypodense lesion without clear delineation in CT) and/or characteristic clinical signs (amaurosis fugax, central retinal artery occlusion or cortical signs such as aphasia, neglect, hemianopsia). Cardiovascular risk factors were defined as described previously. $^{2}$

\section{TOE imaging protocol}

TOE examinations were performed by one experienced senior echocardiographer using a $5 \mathrm{MHz}$ multiplane probe (ATL, HDI 5000, Bothell, USA), recorded on videotape and reviewed offline blinded to the MRI results. Three predefined aortic segments (ie, ascending aorta, aortic arch and descending aorta) were imaged in cross sectional and longitudinal orientation. Evaluation criteria included: (a) maximum aortic wall thickness (AWT) (ie, intima-media thickness at sites free of plaques); (b) number and location of plaques (ie, irregular and circumscribed thickening of the intima with increased echogenicity $\left.{ }^{19}\right)$ per aortic segment, differentiation into low risk (ie, $<4 \mathrm{~mm}$ ) and high risk plaques (ie, $\geqslant 4 \mathrm{~mm}$ in thickness or presence of ulcerations or mobile components) and exact localisation, as derived from a cross sectional view; and (c) detection of aortic thrombi defined as laminated deposition along the intimal surface with variable echogenicity which may be associated with mobile lesions. ${ }^{20} 21$

\section{MR imaging protocol}

All examinations were performed on a $3 \mathrm{~T}$ system (TRIO; Siemens Medical Solutions, Erlangen, Germany) equipped with an 8 channel phased array body coil. Plaque imaging was performed using a T1 weighted rf-spoiled and fat saturated 3D gradient echo sequence. Three dimensional volumes were carefully adapted to cover the aortic arch and the ascending and descending aorta (voxel size $=0.8 \times 1.1 \times 1.1 \mathrm{~mm}^{3}$; field of view $(\mathrm{FOV})=350 \times 252 \mathrm{~mm}^{2}$; echo time $(\mathrm{TE}) /$ repetition time $(\mathrm{TR})=1.7 / 5.1 \mathrm{~ms} ;$ flip angle $\left.=20^{\circ}\right)$. To minimise motion induced blurring, data acquisition was executed using ECG gating with a short diastolic data acquisition window (158 ms) within the cardiac cycle.

Respiration control by navigator gating allowing for data acquisition during free breathing and minimisation of breathing artefacts was performed as described previously. ${ }^{22}$ Retrospectively, ECG gated 2D time resolved (CINE) gradient echo imaging (voxel size $=1.6 \times 2.1 \times 5 \mathrm{~mm}^{3}$; temporal resolution $=26.8 \mathrm{~ms} ; \mathrm{FOV}=400 \times 300 \mathrm{~mm}^{2} ; \mathrm{TE} / \mathrm{TR}=2.0 / 3.4 \mathrm{~ms}$; flip angle $=15^{\circ}$ ) normal to the ascending aorta was performed at end expiration to individually determine the temporal frames within the cardiac cycle with the least vessel motion. The data acquisition window for 3D imaging was then set to match the period with no or only minor aortic motion.

During the MRI examination, data were analysed using a multiplanar reformat tool available on the scanner software (Siemens WIZARD; Siemens Medical Solutions). Consensus reading of two experienced observers was performed to identify plaques $\geqslant 3 \mathrm{~mm}$. To optimise scan and data evaluation efficiency, data acquisition was split into two axial 3D volumes $3.52 \mathrm{~cm}$ thick in order to evaluate the first 3D data volume while the second acquisition was performed.

In the presence of aortic plaques $\geqslant 3 \mathrm{~mm}$ in thickness, contrast agent (gadobenate dimeglumine, Gd-BOPTA chelate, Multihance; Altana Pharma, Konstanz, Germany) was injected as a single dose $(0.1 \mathrm{mmol} / \mathrm{kg}$ body weight, injection rate 3$4 \mathrm{ml} / \mathrm{s}$ ) and time resolved contrast enhanced $3 \mathrm{D}$ MR angiography (tr-CE-MRA) was performed. Imaging parameters included parallel imaging (GRAPPA, acceleration factor $=4^{23}$ ) 
and partial Fourier acquisition along the phase and slice encoding direction (partial Fourier factor $=6 / 8$ ). The combination with view sharing along the temporal domain (TREAT, double update rate of central $\mathrm{k}$-space ${ }^{24}$ ) resulted in a reconstructed voxel size of $1.3 \times 1.9 \times 1.5-1.8 \mathrm{~mm}^{3}$ and an effective (reconstructed) temporal update rate of $2.7-3.0 \mathrm{~s}$ $\left(\mathrm{FOV}=400 \times 400 \mathrm{~mm}^{2} ; \mathrm{TE} / \mathrm{TR}=0.8 / 2.0 \mathrm{~ms} ;\right.$ flip angle $=15-$ $\left.25^{\circ}\right)$. Twenty $\mathrm{T} 1$ weighted $3 \mathrm{D}$ data volumes were acquired in the sagittal oblique or coronal orientation covering the entire thoracic aorta.

To increase the reliability of plaque detection by improved signal-to-noise ratio, $\mathrm{T} 1$ high spatial resolution $3 \mathrm{D}$ plaque characterisation was repeated after administration of contrast agent. Breath held T2 weighted (voxel size $=1.5 \times 2.1 \times 5.0 \mathrm{~mm}^{3}$; FOV $=340 \times 276 \mathrm{~mm}^{2} ; \quad \mathrm{TE} / \mathrm{TR}=86.0 \mathrm{~ms} / 2 \times \mathrm{RR} ; \quad$ flip angle $\left.=90-(160-180)^{\circ}\right)$ and time resolved (CINE) images were acquired at slice positions corresponding to previously detected plaque locations $\geqslant 3 \mathrm{~mm}$.

\section{MR data analysis}

Based on consensus reading, MRI data were evaluated by two experienced readers blinded to patient demographics and TOE data. Image quality of additionally acquired T2 weighted images was categorised as: low = severe blurring of the aortic wall and insufficient black blood saturation, reliable assessment of the vessel wall impossible; moderate $=$ minor blurring of the aortic wall and incomplete black blood saturation, reliable assessment of the vessel wall possible; and high = exact delineation of the aortic wall and complete black blood saturation, assessment possible with a high level of confidence. Aortic plaque was defined as a circumscript thickening of the aortic wall. Maximal extent of plaque involvement was evaluated for each segment of the aorta using all available pre- and post-contrast MR images. The outlets of the great supra-aortic arteries served as landmarks for the description of exact plaque localisation within the 3D data volume. Maximum plaque thickness (AWT) was measured by electronic callipers in the transverse view as the distance between the aortic border and the point of greatest luminal protrusion (see also fig 2). The largest plaque of each aortic segment (ascending, arch and descending) was measured and considered for further evaluation. Aortic thrombi were defined as mobile structures floating within the bloodstream in the transverse view in time resolved CINE imaging with hypointense signal in CINE and T1 images without contrast enhancement.

MR plaque analysis was performed using a multiplanar reformat tool (J-Vision; Tiani Medgraph AG, Innsbruck, Austria) to navigate within the $3 \mathrm{D}$ data set and generate preand post-contrast images of aortic plaques in planes normal to the aorta. For further orientation, the exact location of

Table 1 Baseline characteristics of the study population $(n=74)$

\begin{tabular}{ll}
\hline Characteristic & \\
\hline Age (y) & $58.9(13.4)$ \\
Female sex (n (\%)) & $32(43.2)$ \\
Hypertension (n (\%)) & $53(71.6)$ \\
Diabetes (n (\%)) & $12(16.2)$ \\
Hyperlipidaemia (n (\%)) & $24(32.4)$ \\
Smoking (n (\%)) & $27(36.5)$ \\
Coronary artery disease (n (\%)) & $5(6.8)$ \\
Peripheral artery disease (n (\%)) & $4(5.4)$ \\
History of stroke/transient ischaemic attack (n (\%)) & $17(23.0)$
\end{tabular}

Values are mean (SD). individual cut planes could be visualised within a maximum intensity projection of the arterial phase of the tr-CE-MRA data (fig 1). Image location and angulations were carefully fine tuned by visually comparing anatomical landmarks in reformatted pre- and post-contrast images.

\section{Comparison of TOE and MRI data}

Aortic segments were defined as follows: (1) ascending aorta: segment from the aortic bulb onto the outlet of the brachiocephalic trunk; (2) aortic arch: the following segment including the outlet of the left subclavian artery; (3) descending aorta: segment distal to the outlet of the left subclavian artery. Following a previous study protocol, localisation and morphology of aortic plaques found in TOE and MRI were documented on two separate sketches. ${ }^{25}$ As a result, we obtained from all data recorded by TOE and MR a total of 222 segments from our 74 patients. AWT, absolute number of plaques of any size and especially those $\geqslant 4 \mathrm{~mm}$ thick or with superimposed thrombi, detection of inflammatory wall disease or dissection membrane in each aortic segment were considered for classification of TOE and MRI findings in each patient.

\section{Statistical analysis}

Data are presented as mean (SD) for continuous variables. To detect statistically significant differences between continuous variables, paired t tests were applied. All significance tests were two sided comparing corresponding aortic segments in TOE and MRI and using a significance level of $5 \%$. For comparison of complex aortic plaques found in TOE and MRI, kappa coefficients ( $\kappa$ ) for each aortic segment were calculated. Comparison between TOE and MRI measurements for maximum plaque thickness was evaluated using the Bland and Altman approach ${ }^{26}$ by calculating the mean $(d)$ and SD $\left(\varsigma_{d}\right)$ of the difference. From these data, the limits of agreements $\left(d-2 \varsigma_{d}\right.$ and $+2 \varsigma_{d}$ ) were calculated. All analyses were performed by SPSS statistical package (V.14.0).

\section{RESULTS}

\section{Basic findings}

Baseline demographic data are given in table 1. Distribution of stroke subtypes is shown in fig 1; embolic brain ischaemia was found in 57 of 74 patients (77.0\%). Most noticeably, MRI detected an additional aortic high risk source of brain embolism in $8 / 26(30.8 \%)$ patients with stroke of undetermined aetiology (ie, no conclusive findings in routine diagnostics or TOE). Nineteen of $26(73.1 \%)$ patients with cryptogenic stroke suffered embolic brain ischaemia.

\section{MRI protocol}

In all patients, the entire imaging protocol was successfully performed within 48.9 (12.5) min (range 24-88). Total scan time for high spatial resolution for each $3 \mathrm{D}$ pre- or post-contrast T1 data volume was 6.2 (1.7) min (range 3-12) depending on heart rate and navigator efficiency (30-60\%).

\section{MRI and TOE results: images}

Image quality for both pre- and post-contrast MRI and maximum intensity projections of tr-CE-MRA at maximum arterial contrast is illustrated in comparison with TOE in fig 2. In general, good image quality in T1 weighted sequences was achieved, particularly after application of contrast agent and only minor blurring and ghosting artefacts due to motion were appreciated and obtained in all other patients. Two dimensional 
Figure 2 (A) Hypointense structure protruding into the proximal descending aorta in MR imaging. Time resolved contrast enhanced 3D MR angiography (tCE-MRA) indicates precise plaque localisation. The corresponding transoesophageal echocardiography (TOE) image visualises a mobile aortic thrombus measuring $\geqslant 8 \mathrm{~mm}$ in length (yellow arrow). AWT, aortic wall thickness; CA, contrast agent. (B) Plaque in the descending aorta with hypointense, central core after contrast application compatible with the echogenic core on TOE (yellow arrows). Measurement of maximal aortic wall thickness (AWT) on MRI images using electronic callipers in the transverse view to determine the distance between the aortic border and the point of greatest luminal protrusion is illustrated by the broken yellow lines. CA, contrast agent.
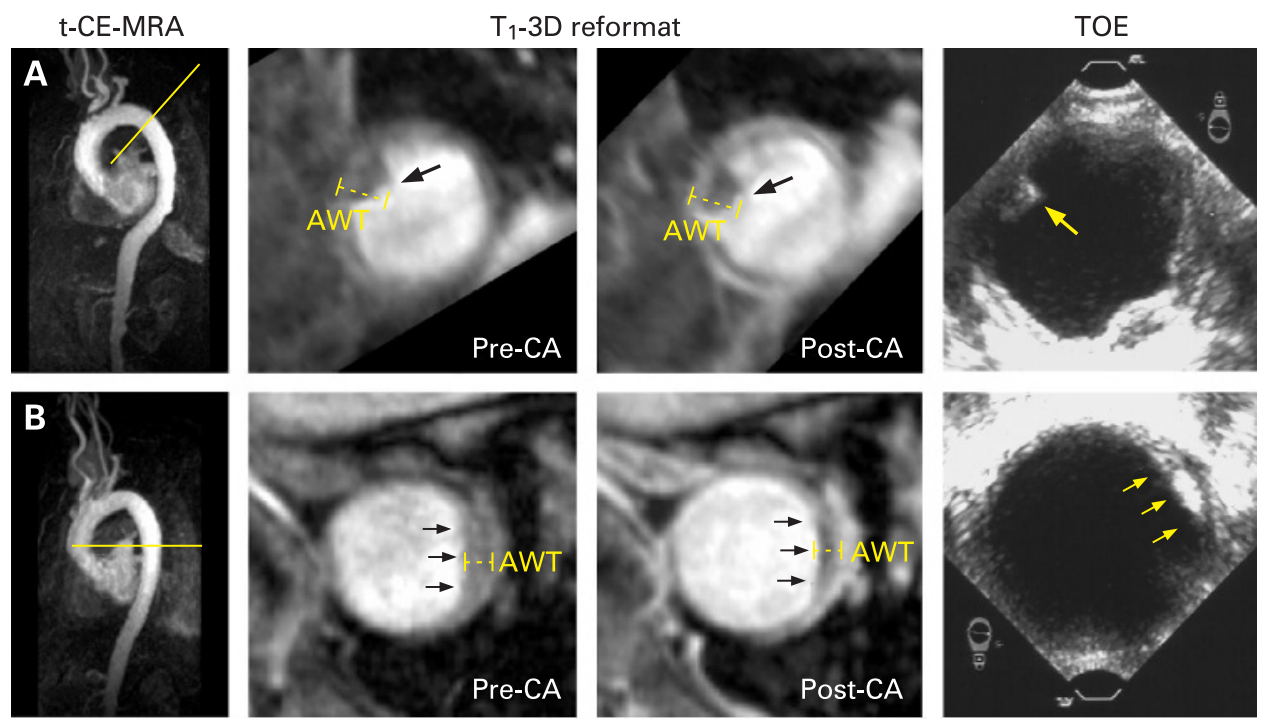

T2 imaging often suffered from unsatisfying image quality (low signal-to-noise ratio, insufficient black blood saturation) and could thus be considered in only part of all examined patients. Overall, 118 T2 weighted measurements of the aorta were performed in 44 patients. Image quality was graded low in 51/ $118(43.2 \%)$, moderate in 35/118 (29.7\%) and high in 32/118 sequences $(27.1 \%)$. T2 imaging proved useful in a patient with type $\mathrm{B}$ aortic dissection (fig 3 ).

\section{Comparison of TOE and MRI data}

The wall of the ascending aorta and aortic arch was not reliably assessable by TOE because of limited visualisation in 11/74 $(14.9 \%)$ and $45 / 74$ patients (60.8\%). Overall, TOE detected aortic high risk plaques in 23/74 (31.1\%) compared with $37 / 74$ $(50 \%)$ patients, as identified by MRI $(p=0.029)$. Also, the absolute number of aortic plaques $\geqslant 4 \mathrm{~mm}$ and thrombi detected by TOE $(n=47)$ was lower than in MRI $(n=74)$ (table 2, fig 4).

Differences in maximum AWT for TOE and MRI were not statistically significant (see table 2). AWT and number of aortic high risk plaques increased from the ascending to the descending aorta. Agreement between MRI and TOE for the detection of aortic segments with plaques $\geqslant 4 \mathrm{~mm}$ or aortic thrombi was moderate to substantial $(0.90>\kappa>0.50)$, as indicated by the kappa coefficients in table 2. A detailed interindividual comparison of findings for all patients is provided by the Bland-Altman plot in fig 5. Results show limited agreement of MRI and TOE measurements in direct comparison. The mean (SD) of the difference was small, whereas the differences between MRI and TOE were relatively wide in comparison with the mean, with the difference increasing with the mean of maximum aortic wall thickness. For the $95 \%$ of the differences within the limits of agreement there was no indication of a systematic error.

Further data analysis revealed that in only four patients MRI overlooked aortic high risk plaques detected by TOE: in two patients the atheroma $\geqslant 4 \mathrm{~mm}$ was located outside the predefined MRI $3 \mathrm{D}$ data volume (ie, in the very proximal ascending or distal descending aorta), in another patient a $4.4 \mathrm{~mm}$ thick plaque in the arch was not detected by MRI and
Figure 3 Patient with graft repair in the ascending aorta $(\mathrm{AAO})$ and type $\mathrm{B}$ dissection in the aortic arch and descending aorta (DAo). The reduced signal in the smaller lumen (black arrows) may falsely be identified as plaque in $\mathrm{T} 1$ MR imaging. In contrast, the dissection flap (white arrows) and dissection lumen can clearly be identified on the dark blood T2 images (upper right) and corresponding transoesophageal echocardiography (TOE) image (lower right). CA, contrast agent; CE-MRA, contrast enhanced 3D MR angiography.
CE-MRA

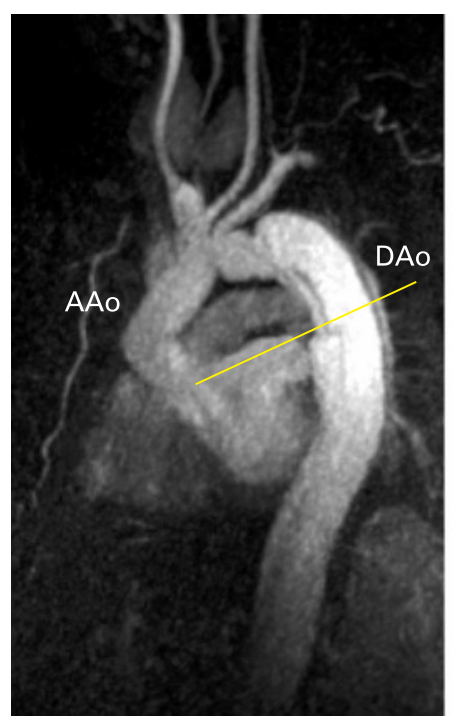

$\mathrm{T}_{1}-3 \mathrm{D}$ reformat
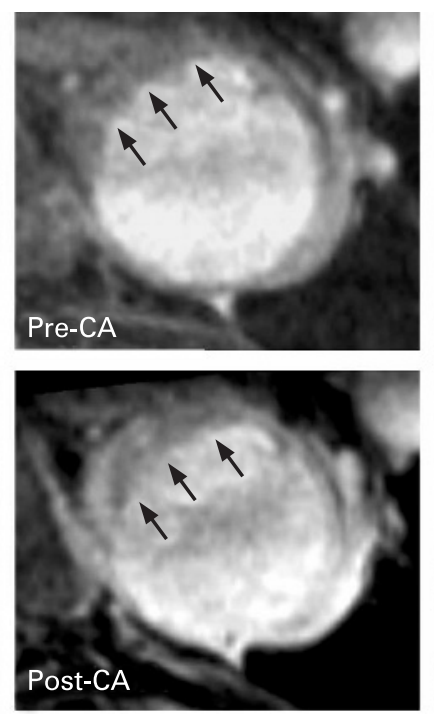

$T_{2}-2 D d b$
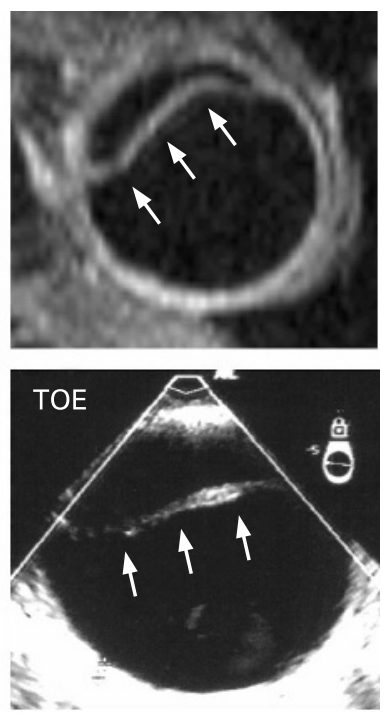
Table 2 Comparison of TOE and MRI findings

\begin{tabular}{llll}
\hline Aortic segment & TOE & MRI & \\
\hline Maximum wall thickness & & & $\mathrm{p}$ Value \\
Ascending aorta $(\mathrm{mm})$ & $2.52(1.01)$ & $2.28(0.89)$ & NS \\
Aortic arch (mm) & $3.36(1.46)$ & $2.83(1.37)$ & NS \\
Descending aorta (mm) & $3.27(1.51)$ & $3.47(1.45)$ & NS \\
\hline No of patients with $\geqslant \mathbf{1}$ complex aortic plaque & & & к coefficient \\
\hline Ascending aorta & 4 & 5 & 0.88 \\
Aortic arch & 10 & 19 & 0.53 \\
Descending aorta & 17 & 36 & 0.52 \\
Absolute No of complex aortic plaques & & & \\
Ascending aorta & 4 & 5 & \\
Aortic arch & 16 & 24 & \\
Descending aorta & 27 & 45 & \\
Summary of all segments & 47 & 74 & \\
\hline
\end{tabular}

Note that the ascending aorta was assessable in 63 , the arch in 30 and the descending aorta in 74 patients by both techniques. Values are mean (SD)

The lower part of the table does not represent an intra-patient correlation. Findings were used to highlight the fact that the incidence of plaques increases from the ascending to the descending aorta and that the absolute number of complex plaques detected by MRI is higher compared with TOE.

TOE, transoesophageal echocardiography.

in the fourth subject measurements of AWT in MRI and TOE were 3.9 and $4.0 \mathrm{~mm}$, respectively. Additional CINE imaging allowed for the detection of a mobile descending aortic thrombus, as confirmed by TOE in one patient. Another mobile thrombus located at the anterior wall of the aortic arch as well as a dissection of the aortic inner curvature not visible in TOE could be clearly identified in the transverse view by MRI CINE imaging. Concordantly, aortic dissection in two patients who had received partial aortic graft repair could be detected by both diagnostics. Inflammatory vessel disease was not observed in any patient.

\section{DISCUSSION}

In our study, plaque detection in the thoracic aorta using high spatial resolution $3 \mathrm{D}$ MRI at $3 \mathrm{~T}$ was successfully demonstrated in 74 acute stroke patients in direct comparison with TOE.

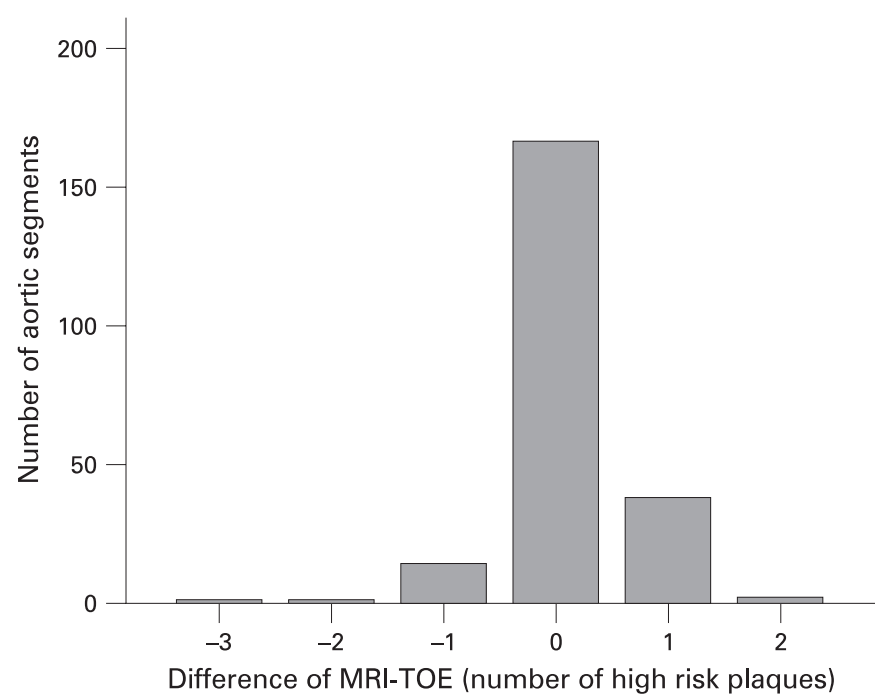

Figure 4 Differences between MRI and transoesophageal echocardiography (TOE) with regard to the absolute number of high risk plaques (ie, $\geqslant 4 \mathrm{~mm}$, superimposed thrombi) in all aortic segments. Negative values indicate a higher number of plaques detected by TOE, whereas positive values indicate higher numbers of plaques were found by MRI.
Although the agreement between MRI and TOE using BlandAltman analysis was poor, further evaluation suggests that MRI may be more reliable than TOE for the detection of high risk aortic diseases in stroke patients: the number of high risk plaques found by MRI was significantly higher compared with TOE which can only partly be explained by the fact that MRI measures all layers of the aortic wall whereas TOE considers intima-media or plaque thickness. The spatial resolution of MRI in this study was not sufficient to fully resolve the thin adventitia which may result in partial volume effects and offer a potential explanation for overestimation. Nevertheless, overestimation was relatively small such that comparing MRI and TOE provided a valid test for evaluation of the reliability of MRI. In contrast with 3D MRI, TOE, the currently best available reference method, failed to reliably assess the aortic

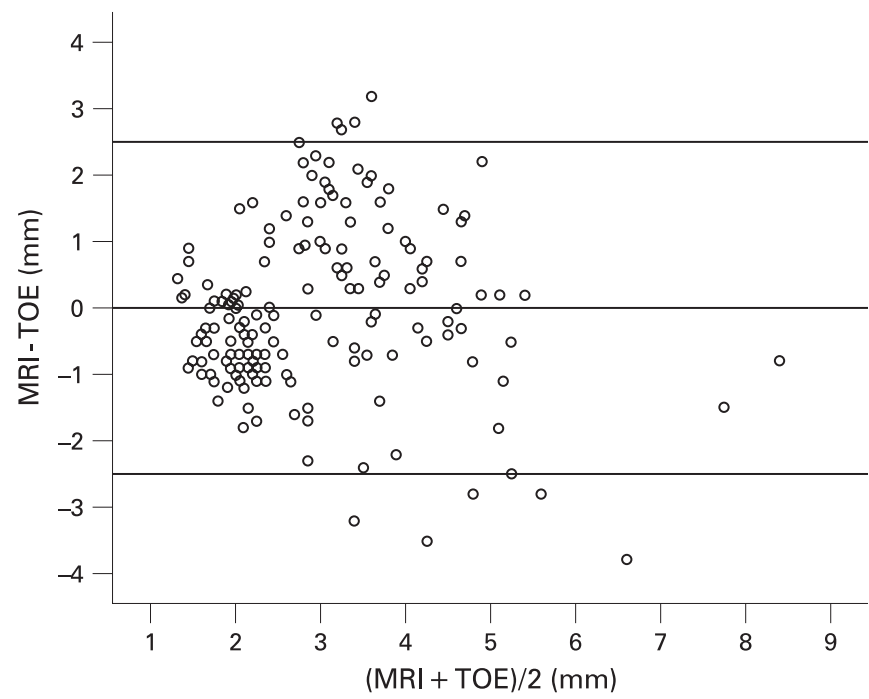

Figure 5 Bland-Altman plot of maximum plaque thickness by transoesophageal echocardiography (TOE) and MRI from matched cross sectional aortic plaque images of the ascending aorta, the aortic arch and the descending aorta for all segments assessable by both TOE and MRI $(\mathrm{n}=167)$. Mean of difference was $-0.05 \mathrm{~mm}$, SD of difference was $1.27 \mathrm{~mm}$ and limits of agreements were mean $-1.96 \times \mathrm{SD}=-2.53 \mathrm{~mm}$ and mean $+1.96 \times \mathrm{SD}=2.44 \mathrm{~mm}$. 
arch because of insufficient visualisation in half of the patients. Therefore, we believe that both the apparent poor agreement of both methods in the Bland-Altman analysis and the higher number of aortic pathologies found by MRI is particularly due to improved aortic coverage and visualisation than to measurement of "false positive" pathologies. Our findings emphasise the need for a method such as 3D MRI. Previous studies confirmed that TOE underestimates the true number of plaques due to limited visualisation of the aortic arch. ${ }^{37} \mathrm{How}^{27}$ ever, a detailed analysis of the number of patients with inappropriate visualisation of the arch in TOE has not been reported.

Most noticeably, additional aortic high risk sources of brain ischaemia were detected by MRI in almost $30 \%$ of patients with stroke of undetermined aetiology after routine diagnostics, including TOE. The significance of these additional MRI findings was confirmed by the high number of embolic strokes in this subgroup. Thus our findings suggest that high field $3 \mathrm{D}$ MRI may increase diagnostic accuracy for the detection of aortic high risk pathologies, especially in patients with cryptogenic stroke. Although the increased risk of cerebral embolisation in patients with severe aortic plaques is well documented, the optimal treatment has not yet been established by prospective and randomised trials and such patients currently receive only antiplatelets and statins. ${ }^{28}$ However, the future value of aortic 3D MRI may increase after the results of the ongoing Aortic arch Related Cerebral Hazard (ARCH) trial comparing aspirin plus clopidogrel versus warfarin for the optimal treatment of aortic high risk plaques.

It is uncertain whether a cut-off value of $\geqslant 4 \mathrm{~mm}$ used to identify high risk plaques on MRI is similar to that on TOE and whether those plaques carry a risk similar to those identified on TOE. Thus, next to the definition of cut-off values for MRI, larger cohorts of stroke patients need to be examined in order to evaluate the role of aortic atherosclerosis in more detail by also detecting those plaques in the ascending aorta and proximal arch that are often overlooked by TOE. High resolution spiral 3D CT yielding complete coverage of the aorta could be an alternative future reference method but next to general disadvantages such as radiation exposure and contrast agent containing iodine, plaque morphology can only to a certain extent been categorised. ${ }^{29}$

To date, only a few in vivo studies have compared MRI and TOE measurements for the evaluation of aortic plaques: Shunk and colleagues ${ }^{30}$ compared TOE with transoesophageal MRI but such MRI coils are not widely available and are also semiinvasive. Two other studies included only a few subjects and MRI was not performed in acute stroke patients. ${ }^{931}$ In contrast with Kutz and colleagues ${ }^{31}$ but consistent with others, ${ }^{9}{ }^{28}$ our results demonstrate both the moderate agreement of AWT and comparable reliability for the detection of aortic high risk plaques for both techniques. In contrast with others, ${ }^{5-7} 91631$ we present a novel MRI protocol at $3 \mathrm{~T}$, providing a $3 \mathrm{D}$ data set with high spatial resolution covering the aorta, including the aortic arch, and evaluate its additional diagnostic impact in acute patients with cryptogenic stroke. The definition of individual scan delays, the short diastolic acquisition window and the technique of respiratory navigator gating provided good diagnostic image quality, even in the aortic arch. Pre- and postcontrast imaging was primarily performed to maximise reliability of aortic plaque detection. Furthermore, application of contrast agent allowed for supplementary time resolved CEMRA, providing additional $3 \mathrm{D}$ information on exact plaque location and thoracic vascular geometry which is especially helpful in patients with aortic aneurysm, type A dissection or Takayasu's arteriitis.

The detailed characterisation of aortic plaque remains challenging: four patients with aortic plaques $\geqslant 4 \mathrm{~mm}$ on $\mathrm{TOE}$ were not identified by MRI because the plaques were located outside the predefined MRI 3D data volume or thickness was minimally underestimated by MRI. Further optimisation therefore includes coverage of the very proximal ascending aorta and a higher spatial resolution (ie, $<1 \mathrm{~mm}$ ). Individually performed T2 weighted 2D and T1 CINE imaging did provide additional information in patients with dissection and aortic thrombi and point towards the importance of both multi-contrast and functional $M R$ imaging. These sequences were not applied routinely because of the currently low image quality in the aortic arch of most patients and the confined scan time in acutely ill patients. Thus future reduction of total scan time and implementation of robust T2 and additional PD weighted pulse sequences into the MRI protocol may ameliorate diagnostic accuracy and plaque characterisation.

\section{SUMMARY}

The utility and quality of an MR imaging protocol for high spatial resolution $3 \mathrm{D}$ examination of aortic high risk pathologies at $3 \mathrm{~T}$ was successfully evaluated in comparison with TOE in acute stroke patients. The reasonable expenditure of time for MRI examination and in particular independence from the breath holding capacities of the imaged subjects allow the implementation of this technique even in acute stroke. The high sensitivity of $3 \mathrm{D}$ MRI for the detection of aortic high risk plaques makes it a promising tool in patients with contraindications for TOE and particularly in those with cryptogenic stroke despite extensive routine diagnostics. However, before this novel MRI technique can be generally recommended, its diagnostic value needs to be evaluated rigorously at various imaging sites.

Funding: MM is supported by the Deutsche Forschungsgemeinschaft (DFG), grant No MA 2383/3-1 and the Bundesministerium für Bildung und Forschung (BMBF), grant No $01 E V 0706$.

\section{Competing interests: None.}

Ethics approval: The study was approved by the local ethics committee.

\section{REFERENCES}

1. Reynolds HR, Tunick PA, Kronzon I. Role of transesophageal echocardiography in the evaluation of patients with stroke. Curr Opin Cardiol 2003;18:340-5.

2. Harloff A, Handke M, Reinhard M, et al. Therapeutic strategies after examination by transesophageal echocardiography in 503 patients with ischemic stroke. Stroke 2006;37:859-64.

3. Amarenco $\mathbf{P}$, Cohen A, Tzourio $\mathrm{C}$, et al. Atherosclerotic disease of the aortic arch and the risk of ischemic stroke. N Engl J Med 1994;331:1474-9.

4. Daniel WG, Erbel R, Kasper W, et al. Safety of transesophageal echocardiography. A multicenter survey of 10,419 examinations. Circulation 1991;83:817-21.

5. Toussaint JF, LaMuraglia GM, Southern JF, et al. Magnetic resonance images lipid, fibrous, calcified, hemorrhagic, and thrombotic components of human atherosclerosis in vivo. Circulation 1996;94:932-8.

6. Saam T, Ferguson MS, Yarnykh VL, et al. Quantitative evaluation of carotid plaque composition by in vivo MRI. Arterioscler Thromb Vasc Biol 2005;25:234-9.

7. Cai J, Hatsukami TS, Ferguson MS, et al. In vivo quantitative measurement of intact fibrous cap and lipid-rich necrotic core size in atherosclerotic carotid plaque: comparison of high-resolution, contrast-enhanced magnetic resonance imaging and histology. Circulation 2005;112:3437-44.

8. Koops A, Ittrich $\mathrm{H}$, Petri S, et al. Multicontrast-weighted magnetic resonance imaging of atherosclerotic plaques at 3.0 and 1.5 Tesla: ex-vivo comparison with histopathologic correlation. Eur Radiol 2007;17:279-86.

9. Fayad ZA, Nahar T, Fallon JT, et al. In vivo magnetic resonance evaluation of atherosclerotic plaques in the human thoracic aorta: a comparison with transesophageal echocardiography. Circulation 2000;101:2503-9.

10. Kramer CM, Cerilli LA, Hagspiel K, et al. Magnetic resonance imaging identifies the fibrous cap in atherosclerotic abdominal aortic aneurysm. Circulation 2004;109:1016-21. 
11. Lima JA, Desai MY, Steen H, et al. Statin-induced cholesterol lowering and plaque regression after 6 months of magnetic resonance imaging-monitored therapy. Circulation 2004;110:2336-41

12. Yonemura A, Momiyama Y, Fayad ZA, et al. Effect of lipid-lowering therapy with atorvastatin on atherosclerotic aortic plaques detected by noninvasive magnetic resonance imaging. J Am Coll Cardiol 2005;45:733-42.

13. Wentzel JJ, Corti R, Fayad ZA, et al. Does shear stress modulate both plaque progression and regression in the thoracic aorta? Human study using serial magnetic resonance imaging. J Am Coll Cardiol 2005;45:846-54.

14. Steen H, Warren WP, Desai M, et al. Combined transesophageal and surface MRI provides optimal imaging in aortic atherosclerosis. J Cardiovasc Magn Reson 2004;6:909-16.

15. Barkhausen J, Ebert W, Heyer C, et al. Detection of atherosclerotic plaque with Gadofluorine-enhanced magnetic resonance imaging. Circulation 2003;108:605-9.

16. Corti R, Fuster V, Fayad ZA, et al. Effects of aggressive versus conventional lipidlowering therapy by simvastatin on human atherosclerotic lesions: a prospective randomized, double-blind trial with high-resolution magnetic resonance imaging. J Am Coll Cardiol 2005:46:106-12.

17. Wiesmann F, Szimtenings M, Frydrychowicz A, et al. High-resolution MRI with cardiac and respiratory gating allows for accurate in vivo atherosclerotic plaque visualization in the murine aortic arch. Magn Reson Med 2003:50:69-74.

18. Adams HP Jr, Bendixen BH, Kappelle LJ, et al. Classification of subtype of acute ischemic stroke. Definitions for use in a multicenter clinical trial. TOAST. Trial of Org 10172 in Acute Stroke Treatment. Stroke 1993;24:35-41.

19. Kallikazaros IE, Tsioufis CP, Stefanadis Cl, et al. Closed relation between carotid and ascending aortic atherosclerosis in cardiac patients. Circulation 2000;102(Suppl)|II263-8.

20. Cohen A, Tzourio C, Bertrand B, et al. Aortic plaque morphology and vascular events: a follow-up study in patients with ischemic stroke. FAPS Investigators. French Study of Aortic Plaques in Stroke. Circulation 1997;96:3838-41.
21. Karalis DG, Chandrasekaran K, Victor MF, et al. Recognition and embolic potential of intraaortic atherosclerotic debris. J Am Coll Cardiol 1991:17:73-8.

22. MarkI M, Harloff A, Bley $\mathrm{T}$, et al. Time resolved 3D MR velocity mapping at $3 \mathrm{~T}$ improved navigator gated assessment of vascular anatomy and blood flow. J Magn Reson Imaging 2007;25:824-31.

23. Griswold MA, Jakob PM, Heidemann RM, et al. Generalized autocalibrating partially parallel acquisitions (GRAPPA). Magn Reson Med 2002:47:1202-10.

24. Fink C, Ley S, Kroeker R, et al. Time-resolved contrast-enhanced three-dimensional magnetic resonance angiography of the chest: combination of parallel imaging with view sharing (TREAT). Invest Radiol 2005;40:40-8.

25. Weinberger J, Azhar S, Danisi F, et al. A new noninvasive technique for imaging atherosclerotic plaque in the aortic arch of stroke patients by transcutaneous realtime B-mode ultrasonography: an initial report. Stroke 1998;29:673-6.

26. Bland JM, Altman DG. Statistical methods for assessing agreement between two methods of clinical measurement. Lancet 1986;1:307-10.

27. Joffe II, Jacobs LE, Lampert C, et al. Atherosclerotic disease of the aortic arch and the risk of ischemic stroke. N Engl J Med 1995;332:1237-8.

28. Kronzon I, Tunick PA. Aortic atherosclerotic disease and stroke. Circulation 2006;114:63-75.

29. Pham PH, Rao DS, Vasunilashorn F, et al. Computed tomography calcium quantification as a measure of atherosclerotic plaque morphology and stability. Invest Radiol 2006:41:674-80.

30. Shunk KA, Garot J, Atalar E, et al. Transesophageal magnetic resonance imaging of the aortic arch and descending thoracic aorta in patients with aortic atherosclerosis. J Am Coll Cardiol 2001:37:2031-5.

31. Kutz SM, Lee VS, Tunick PA, et al. Atheromas of the thoracic aorta: A comparison of transesophageal echocardiography and breath-hold gadolinium-enhanced 3dimensional magnetic resonance angiography. J Am Soc Echocardiogr 1999;12:853-

\section{Submit an eletter, and join the debate}

eLetters are a fast and convenient way to register your opinion on topical and contentious medical issues. You can find the "submit a response" link alongside the abstract, full text and PDF versions of all our articles. We aim to publish swiftly, and your comments will be emailed directly to the author of the original article to allow them to respond. eLetters are a great way of participating in important clinical debates, so make sure your voice is heard. 